\title{
Analysis of Compensation Network in a Correlated- based Channel using Angle of Arrivals
}

\author{
Affum Emmanuel Ampoma \\ Centre for RFIC and System Technology, \\ School of Communication and Information Engineering, \\ University of Electronics Science and Technology of China, \\ Chengdu 611731, P.R. China
}

\author{
Obour Agyekum Kwame O.-B \\ School of Information and Communication Engineering, \\ University of Electronics Science and Technology of China, \\ Chengdu 611731, P.R. China
}

\author{
Paul Oswald Kwasi Anane \\ School of Information and Communication Engineering, \\ University of Electronics Science and Technology of China, \\ Chengdu 611731, \\ P.R. China
}

\author{
Maxwell Oppong Afriyie \\ School of Information and Communication Engineering, \\ University of Electronics Science and Technology of China \\ Chengdu 611731, P.R. China
}

\begin{abstract}
We explore combined effect of spatial correlation and mutual coupling matrix, and its subsequent effects on performance of multiple input multiple output (MIMO) systems After the decoupling process. We will also look at a correlation based stochastic channel model with the linear antenna arrays as the signal source. For the purpose of understanding, it is assumed that fading is correlated at both transmitter and receiver sides, in spite of the fact that the decoupling network enhances isolation between Receiving antenna array. In this paper, we model the transmit the antenna array in CST Microwave Studio, as a uniform linear Array with monopoles as antenna elements. On the receiving side, the scattering parameters of the coupled and decoupled monopole Array are measured in an anechoic chamber. The theoretical analysis and simulation results show the joint dependency of the system capacity on an angle of arrival (AoA) and antenna element spacing, with enhanced system performance at reduced AoAs with Increased antenna element separation. Consequently, essential benefits of MIMO system performance can be achieved with an efficient decoupling network while boosting the signal sources by adding further antenna elements.
\end{abstract}

Keywords-Angle of arrival (AoA); channel correlation; decoupling network; mutual coupling; MIMO

\section{INTRODUCTION}

One of the challenges of MIMO antenna design is the task of enhancing the isolation between ports nearly located within restricted space in the mobile handset. This is because of the way the array elements have to be contained in a reduced volume, which brings about substantial pattern/spatial correlation and Strong mutual coupling effect between the elements. It is the common conclusion that mutual coupling influences the performance of antenna arrays, as the increase in correlation Restricts the channel capacity. Moreover, if mutual coupling is solid, a massive portion of the power fed into one port will be coupled to the other port rather than radiating to free space; consequently diminishing the signalto-noise ratio, radiation Sufficiency and channel capacity. Some of works have investigated the effect of mutual coupling on the performance of communication system [1]-[10]. For this reason, building a successful decoupling technique to balance the performance degradation in MIMO antennas by mutual coupling effects has attracted the attention of the academic society recently.

In [11] researchers separates decoupling strategies into four classes: 1) Eigen-mode Decomposition Scheme: Its guideline is to diagonalize the scattering matrix of a compact array using $90^{\circ}$ and or $180^{\circ}$ [12]-[16]. 2). The Inserted Component Scheme: It works on the concept of inserting a section of transmission-line between the coupled antenna ports [17]-[21]. 3). Artificial Structure Decoupling Scheme: This method uses sub-wavelength EM structures such as electromagnetic band gap (EBG) structure [22], defected ground structures (DGS) [23], and magnetic meta-materials [24], [25]. 4). Coupled Resonator Decoupling Scheme: This method was proposed for the first time in 2014, and has the concept of decoupling pair of coupled elements using coupled resonators [11] and [26]-[29].

Various works have examined the impact of spatial correlation on the performance of communication systems by means of experimentation [30], [31], modeling [32], [33] and theoretical analysis [34]-[40]. Numerous works have characterized the effect of channel correlation in the performance and capacity of the wireless channels by mathematical analysis [34], [35], further focusing on linear receivers [36], [37], [40] using mainly random matrix theory [41], [42], [43]. In [33] and [44]-[48] precoder designs Specifically tailored for correlated channels are derived. An interesting channel model with transmitting correlation, based on the angular spread of the transmits antenna elements emission, was shown by the authors of [33], [49].

The above discussions focus on studying the influence of antenna separation on a set number of antennas and effects on The communication performance. The purpose of compensation or decoupling network, however, is to enhance the isolation between ports jointly located within restricted space in mobile handset. It is, therefore, reasonable to investigate the combined effect of spatial correlation and 
mutual coupling matrix on correlation-based stochastic channel coefficients, and subsequent effects on system capacity and transmit diversity After the decoupling process. To recognize user equipment (UE) channels and enhance channel estimation, the correlation channel model introduces angle of arrivals (AoAs).

For this reason, we investigate the system performance of a user equipment (UE) channels at different orientations for the uniform linear antenna arrays at diverse antenna separations, while increasing the signal sources by adding the further antenna elements. For the purpose of demonstrating the effectiveness of the research, we utilized a prototype of two-element compensation network with insertion losses between input and Output ports better than $11 \mathrm{~dB}$. Using the spatial correlation model [33] and incorporating the effects of mutual coupling matrix before and after the decoupling process, the results reveal different system performances for the user equipment (UE) channels at reduced AoAs and antenna physical separation while adding more elements.

The paper is organized as follows: Section II presents the formulation of operating matrix and the design of the compensation network. Part III focuses on the system model. Analytical results and discussions are presented in Section IV. Finally, we give concluding remarks in Section V.

\section{OPERATING MATRIX AND DESIGN OF COMPENSATION NETWORK}

The Operating pattern for two-element receiving array for the remittance network is expressed as [54]

$$
\left[\begin{array}{c}
U_{1} \\
U_{2}
\end{array}\right]=\left[\begin{array}{cc}
1 & -\frac{Z_{12}}{Z_{L}} \\
-\frac{Z_{21}}{Z_{L}} & 1
\end{array}\right]\left[\begin{array}{l}
V_{1} \\
V_{2}
\end{array}\right]=\left[\begin{array}{l}
V_{1}-\frac{Z_{12}}{Z_{L}} V_{2} \\
V_{2}-\frac{Z_{21}}{Z_{L}} V_{1}
\end{array}\right]
$$

Where $V_{1}$ and $V_{2}$ are the coupled voltages and the inputs to the network from the monopole terminals and the output voltages are $U_{1}$ and $U_{2}$, also known as the compensation voltages. The compensation network is designed using a power divider of unequal power-dividing ratio with no active circuit elements to minimize extra circuit noise and two ratrace couplers. The power divider has three transmission lines ( $Z_{A}, Z_{B}$ and $Z_{C}$ ), each having impedance of $\sqrt{2} Z_{o}$, where $Z_{\circ}$ is the system's characteristic impedance, but unequal electrical Lengths $\phi, \Psi$ and $\theta$ [55]. The electrical length $\phi$ can be defined as $\phi=\cos ^{-1}\left(\left|Z_{M} / Z_{L}\right|\right)$, whereas $\Psi$ and $\theta$ are $90^{\circ}$ and $\left(90^{\circ}+\phi\right)$ respectively and $Z_{M}$ representing the mutual impedance. We fabricate the circuit by using the substrate FR4 with dielectric constant 4.8 at operating frequency of $2.4 \mathrm{GHz}$ as shown in Figure 1. The measured insertion losses between input and output ports of the decoupling network are shown in Figure 2.

\section{SYSTEM MODEL}

In this paper, we examine the theoretical performance of MIMO system in the correlation-based stochastic channel Models with the decoupling network. It has been accounted for in [50] that the correlation-based stochastic channel models could be applied to cases that the user equipment (UE) with multiple antennas work at millimeter wave, in any case, we experiment the performance of the MIMO systems at 2.4 $\mathrm{GHz}$. For the purpose of understanding, it is assumed fading is correlated at both transmitter and receiver sides, in spite of the fact that, the decoupling network enhances isolation between antenna arrays at the user equipment (UE). When the linear the antenna array is assumed, the steering matrix $R_{k}$ is expressed as [50], [51]

$$
R_{k}=\frac{1}{D_{k}}\left[a\left(\theta_{k, 1}\right), a\left(\theta_{k, 2}\right), \ldots, a\left(\theta_{k, \mathrm{D}_{k}}\right)\right]
$$

$$
a\left(\theta_{k, \mathrm{i}}\right)=\left[1, e^{(j 2 \pi d / \lambda) \sin \theta_{k, \mathrm{i}}}, \ldots, e^{(j 2 \pi d(N-1) / \lambda) \sin \theta_{k, \mathrm{i}}}\right]^{T}
$$

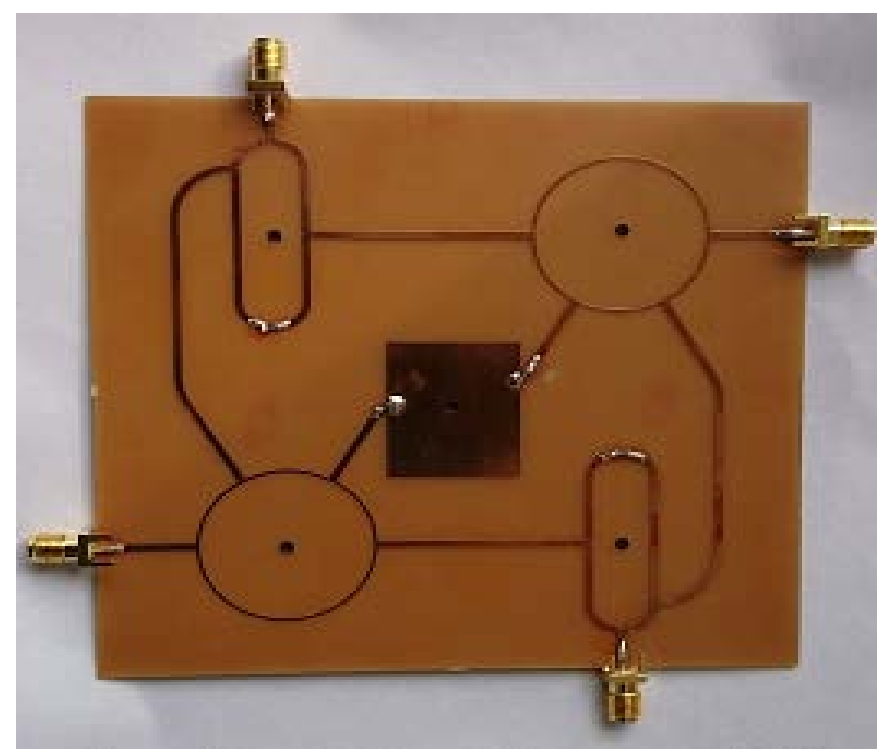

Fig. 1. Photograph of the fabricated inserted decoupling network

Where $d$ is the distance between the adjacent antennas, $\lambda$ is the carrier wavelength and $N$ the number of elements. Incorporating mutual coupling the channel vector UE can be written as follows

$$
h_{k}=Z R_{k} v_{k}, k=1,2, \ldots, k
$$

Where, $Z \in \mathfrak{M}^{N \times N}$ represents the mutual coupling matrix, $R_{k} \in \mathfrak{M}^{N \times D_{k}}$ denotes the steering matrix containing $D_{k}$ steering vectors of the receiver array, $v_{k} \sim \mathfrak{M N}\left(0, I_{D_{k}}\right)$. The mutual coupling matrix is defined as [51], [52] 


$$
Z=\left(Z_{A}+Z_{L}\right)\left(\Gamma+Z_{L} I\right)^{-1}
$$

with

$$
\Gamma=\left[\begin{array}{ccccc}
Z_{A} & Z_{m} & 0 & \cdots & 0 \\
Z_{m} & Z_{A} & Z_{m} & \cdots & 0 \\
0 & Z_{m} & Z_{A} & \cdots & 0 \\
\vdots & \vdots & \ddots & \ddots & \vdots \\
0 & 0 & \cdots & Z_{m} & Z_{A}
\end{array}\right]
$$

Where, $\boldsymbol{Z}_{A}, \boldsymbol{Z}_{L}, \boldsymbol{Z}_{m}$ are the antenna impedance, load impedance and mutual impedance respectively. For theoretical approximation of bilateral coupling (4) we assume that $Z_{A}$ and $\boldsymbol{Z}_{L}$ have a value of $50 \Omega$ each. $\boldsymbol{Z}_{m}$ is calculated using the EMF method [7] using $d$.

\section{A. S-parameter Based Mutual Coupling}

Regarding scattering parameters the mutual coupling the Matrix in (5) of the linear array is expressed as [53]

$$
Z_{t}=\left(I+S_{t}\right)\left(I-S_{t}\right)^{-1} * Z_{o}
$$

Where $Z_{\circ}$ represent the reference antenna impedance, and $S_{t} \in \mathfrak{M}^{N \times N}$ is the S-parameter matrix of the antenna array. The voltage and current on the $m$ - the antenna element are given as

$$
v_{m}=\sqrt{Z_{o}}\left(a_{m}+b_{m}\right) \text { and } i_{m}=\frac{1}{\sqrt{Z_{0}}}\left(a_{m}-b_{m}\right)
$$

Where vectors $a$ and $b$, are the complex envelopes of the inward-propagating and outward-propagating waves from the antenna elements respectively. In this paper, we model the transmit antenna array in CST Microwave Studio, as a uniform linear array with monopoles as antenna elements. For the receiving antenna array, the scattering parameters of the coupled and decoupled monopole array are measured in an anechoic chamber.

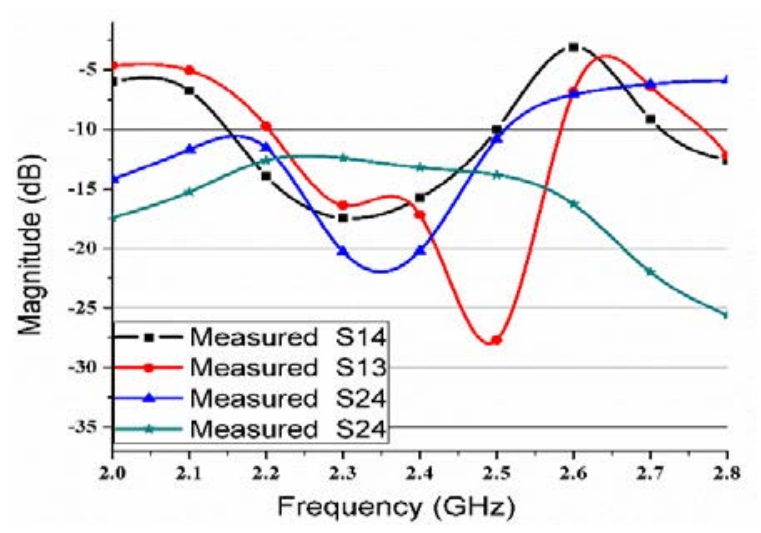

Fig. 2. Measured insertion losses between input and output ports of the Decoupling Network

\section{NUMERICAL RESULTS}

This section illustrates the analytical performance and results according to the modeling of mutual coupling matrix at the Receiving side. The simulation assumes a uniform linear array With monopoles as antenna elements at the source. The compact antenna array consists of two parallel monopoles operating at $2.4 \mathrm{GHz}$ and placed on a metallic ground. The monopoles have the length of $30 \mathrm{~mm}$, a radius of $0.5 \mathrm{~mm}$ and fixed element separation of $\lambda / 8$. The S-parameters for the receiving array are determined in two different conditions. Firstly, the scattering parameters of the coupled monopole array are measured in order to determine the coupled voltages $\left(V_{1}\right.$ and $V_{2}$ ) and coupling matrix. Secondly, the monopole antennas in the array are connected to the decoupling network through equivalent length coaxial links, and scattering parameters of the output ports of the decoupling network are measured to determine the coupling matrix for the compensated voltages ( $U_{1}$ and $U_{2}$ ).

The simulated channel is according to the channel model (1)-(6) and the angle of arrival is between $0^{\circ}$ and $3600^{\circ}$ For the purpose of demonstrating the effectiveness of the decoupling network, there are three different types of voltages listed in Table I. The last row in Table I is a ratio of the voltage obtained with monopole $\mathrm{B}$ to the voltage achieved with monopole A. It can be seen that the ratio of the compensated 
voltage is very close to the uncoupled voltages, demonstrating that the compensated voltages have successfully taken off from the coupling effect.

\section{A. Fixed Number of Antennas- Traditional View}

We investigate the effect of antenna spacing on performance according to coupling matrix model at the receiving side. For reasons of reference, first, we analyze the performance of the uncorrelated channel when the receiving antenna array are closely spaced with a separation of $\lambda / 8$.

We do this for an angle of arrival at the receiving end as $360^{\circ}$ for SNR of $10 \mathrm{~dB}$, to illustrate the behavior of system performance regarding dependency between antenna spacing and average capacity. Figure 3 reveals that capacity of the decoupled receiving array is statistically better than that of the coupled receiving array. In order words, the results demonstrate the promising potentials of an efficient decoupling scheme in a correlated-based stochastic channel. However, system performance is restricted to antenna element spacing. With the decoupling network at the receiving end, therefore, results show the performance benefits that can be achieved by increasing the separation between antenna elements.

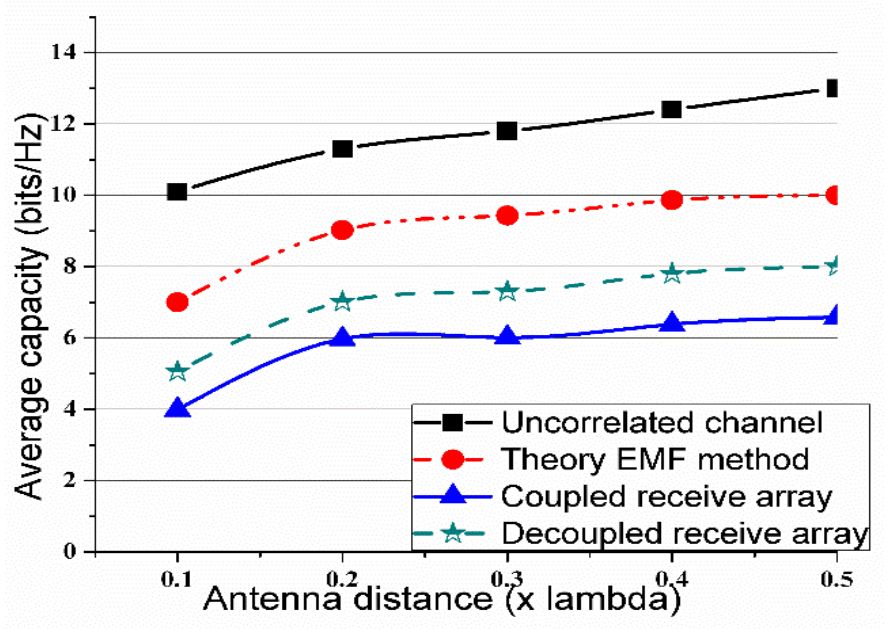

Fig. 3. Average capacity vs. antenna spacing $d$ with $\mathrm{SNR}=10 \mathrm{~dB}, \mathrm{AoA}=$ $360^{\circ}$

\section{B. Effects of AoA on System Performance}

We now move forward to investigate the behavior of channel capacity with different AoAs at various antenna Elements separations at the transmitter. In Figure 4 we compare the performances for $2 \times 2$ systems for AoAs of $60^{\circ}$ and $360^{\circ}$ at element separation of at $d t=\lambda / 8, \lambda / 4, \lambda / 2$. We repeat the process with increasing number of transmit antennas for AoAs Of $90^{\circ}$ and $120^{\circ}$ in figure 5 for $4 \times 2$ MIMO system. The graphs reveal that system performance at reduced AoAs with the increase antenna element separation outperformed that with increased AoA and reduced antenna separation. The results also demonstrate the dependency of channel capacity on an antenna separation. We note that reducing the distance between antenna elements affect system performance, however, the larger number of antenna elements at the signal source improves system performance. It is interesting to observe in Figure 5 that even though the channel is modeled with spatial correlation and mutual coupling at both ends, the performance for AoA of $60^{\circ}$ at $\lambda / 2$ a uncorrelated Mutual coupling at $\mathrm{SNR}=10 \mathrm{~dB}$. The result demonstrates the advantage of decoupling system in a correlated channel with reduced AoA

\section{Transmit Diversity}

For larger performance gain of almost $20 \mathrm{~dB}$ at error rate of $10^{-2}$ figure 6 indicates no match between decoupled and coupled receive arrays for AoA $=360^{\circ}$. However, a close observation in Figure 7 reveals an improvement in the BPSK performance. In spite of the fact that there was little change in the QPSK performance, we take note of that after an increase of $10 \mathrm{~dB}$, the performance for coupled array with both modulations remains Nearly the same. In general, our analysis indicates a close match between QPSK and BPSK diversity performances under both conditions. We include theoretical performance based on the EMF method which outperformed the simulation results.

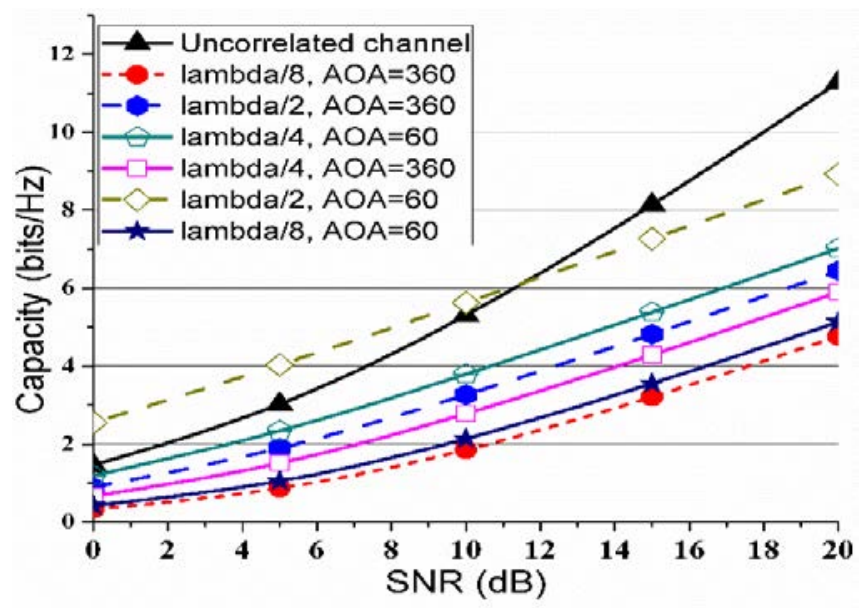

Fig. 4. Capacity vs. SNR for decoupling array for $2 \times 2$ MIMO at different AoAs (degrees) and antenna separation

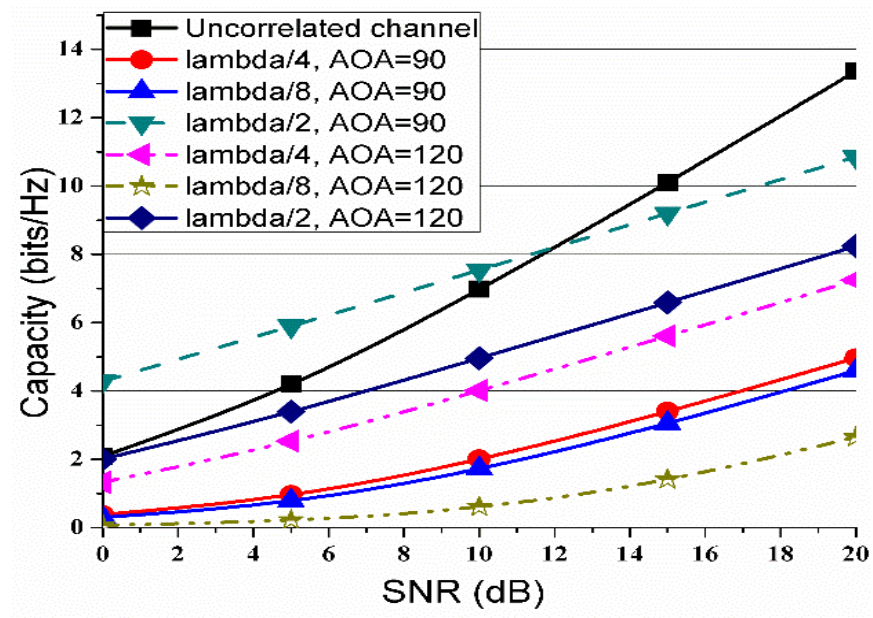

Fig. 5. Capacity vs. SNR for decoupling array for $4 \times 2$ MIMO at different AoAs and antenna element separation 


\section{CONCLUSION}

In this paper, we have analytically explored the performance of decoupling network in the correlation-based stochastic model With uniform linear array at the transmitter. Performance evaluation indicates the joint dependency of system capacity on the angle, angle of arrival and antenna separation. Results show that an important benefit of system performance can be achieved for reduced AoA with increase antenna element separation at the transmitter. Our analysis demonstrates the promising potentials when mutual coupling matrix is modeled with efficient Decoupling network for a compact antenna array. Further work can be carried out towards investigating the performance the behavior of rectangular and circular arrays with decoupling network for MIMO spatially correlated transmitters.

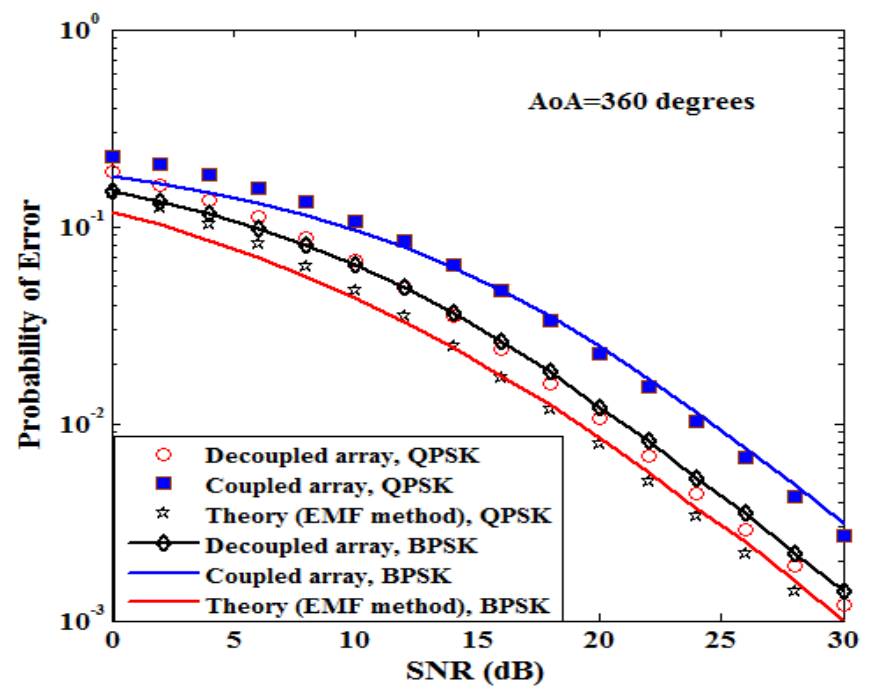

Fig. 6. Transmit diversity for decoupled and coupled array in correlated based stochastic model with linear array

\begin{tabular}{|c|c|c|c|c|}
\hline \multicolumn{5}{|c|}{ TABLE I. } \\
\hline & & $\begin{array}{l}\text { Uncoupled } \\
\text { Voltages } \\
\text { (reference) }\end{array}$ & $\begin{array}{l}\text { Coupled } \\
\text { voltages }\end{array}$ & $\begin{array}{l}\text { Compensated } \\
\text { voltages }\end{array}$ \\
\hline \multirow{2}{*}{$\begin{array}{l}\varangle \\
0 \\
0 \\
0.0 \\
0 \\
\Sigma\end{array}$} & $\begin{array}{l}\text { mag } \\
(\mathrm{mV})\end{array}$ & 16.64 & 12.4 & 11.55 \\
\hline & $\begin{array}{l}\text { angle } \\
\left({ }^{\circ}\right)\end{array}$ & -160.64 & -166.67 & 34.967 \\
\hline \multirow{2}{*}{$\begin{array}{l}n \\
0 \\
0 \\
0 \\
0 \\
0 \\
\Sigma\end{array}$} & $\begin{array}{l}\operatorname{mag} \\
(\mathrm{mV})\end{array}$ & 16.54 & 15.42 & 12.30 \\
\hline & $\begin{array}{l}\text { angle } \\
\left({ }^{\circ}\right)\end{array}$ & -139.56 & -141.46 & 55.16 \\
\hline \multirow{2}{*}{$\mathrm{B} / \mathrm{A}$} & $\begin{array}{l}\text { mag } \\
(\mathrm{mV})\end{array}$ & 0.9939 & 1.2199 & 1.065 \\
\hline & $\begin{array}{l}\text { angle } \\
\left({ }^{\circ}\right)\end{array}$ & 21.08 & 25.208 & 20.193 \\
\hline
\end{tabular}

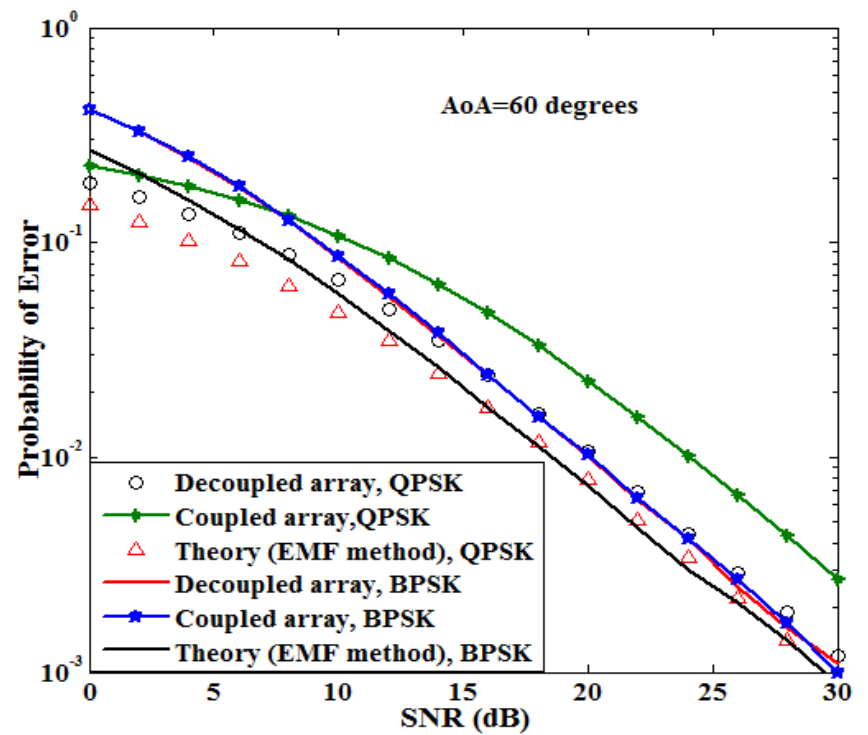

Fig. 7. Transmit diversity for decoupled and coupled array in correlatedbased stochastic model with the linear array

\section{ACKNOWLEDGMENT}

The authors are grateful to all the members of Center for RFIC and System Technology and Multidimensional Signal Processing Laboratory (MSPL), School of Communication and Information Engineering, University of Electronic Science and Technology of China for relevant advice and discussion to this

\section{REFERENCES}

[1] I. J. Gupta and A. A. Ksienski, "Effect of Mutual Coupling on the Performance of Adaptive Arrays," IEEE Transactions on Antennas and Propagation, AP-31, 5, September 1983, pp. 785-791.

[2] T. Svantesson. "The Effects of Mutual Coupling Using a Linear Array of Thin Dipoles of Finite Length". In Proc. 8th IEEE SSAP, pages 232235, Portland, September 1998.

[3] T. Svantesson and A. Ranheim, "Mutual coupling effects on the capacity of multi-element antenna systems," in Proc. IEEE In Conf.: Acoustics, Speech, and Signal Processing, vol. 4, Salt Lake City, UT, May 7-11, 2001, pp. 2485-2488.

[4] Griffith, K.A.; Gupta, I.J., 'Effect of mutual Coupling on the performance of GPS AJ Antennas” EEE/ION, Pages: 871 - 877, 2008.

[5] Hao Yuan, Hirasawa, K. and Yimin Zhang, "The mutual coupling and diffraction effects on the performance of a CMA adaptive array" IEEE Transactions on Vehicular Technology, volume: 47, Issue: 3, Pages: 728 $-736,1998$.

[6] S. Lu, H. T. Hui, M. E. Bialkowski, X. Liu, H. S. Lui, and N. V.Shuley, "Effects of antenna mutual coupling on the performance of MIMO systems," in Proc 29th Symposium on Information Theory, pp. 29452948.

[7] C. A. Balanis, Antenna Theory: Analysis and Design, 3rd Edition.

[8] A. A. Abouda and S. G. Haggman, "Effect of mutual coupling on capacity of MIMO wireless channels in High SNR," Progress in Electromagnetics Research, vol. 65, p. 27-40, 2006.

[9] B. Clerckx, C. Craeye, D. Vanhoenacker-Janvier, and C. Oestges, "Impact of antenna coupling on 2 x 2 MIMO communications," IEEE Trans. Veh. Technol., vol. 56, no. 3, pp. 1009-1018, May 2007,

[10] T. Ratnarajah and A. Manikas, "An H-infinity approach to mitigate the effects of array uncertainties on the MUSIC Algorithm,” IEEE Signal Process. Lett., vol. 5, no. 7, pp. 185-188, Jul. 1998. 
[11] L. Zhao, L. K. Yeung, and K.-L. Wu, "A coupled resonator decoupling network for two-element compact antenna arrays in mobile terminals, ”IEEE Trans. Antennas Propag., vol. 62, no. 5, pp. 2767-2776, May 2014.

[12] J. C. Coetzee and Y. Yu, "Port decoupling for small arrays by means of an eigenmode feed network,” IEEE Trans. Antennas Propag., vol. 6, pp. 1587-1593, Jun. 2008.

[13] S. Zuo, Y.-Z. Yin, Y. Zhang, W.-J.Wu, and J.-J. Xie, "Eigenmode decoupling for MIMO loop-antenna based on 180 coupler," Progr. Electromagn. Res. Lett., vol. 26, pp. 11-20, 2011.

[14] S. K. Chaudhury, H. J. Chaloupka, and A. Ziroff, "Multiport antenna Systems for MIMO and Diversity,” EUCAP, Barcelona, Spain, Apr. 2010.

[15] C. Volmer, J. Weber, R. Stephan, K. Blau, and M. A. Hein, “An eigen analysis of compact antenna arrays and its application to port decoupling,” IEEE Trans. Antennas Propag., vol. 56, no. 2, pp. 360-370, Feb. 2008.

[16] L. K. Yeung and Y. E. Wang, "Mode-based beamforming arrays for miniaturized platforms," IEEE Trans. Microw. Theory Tech., vol. 57, no. 1, pp. 45-52, Jan. 2009.

[17] J. B. Andersen and H. H. Rasmussen, "Decoupling and descattering networks for antennas,” IEEE Trans. Antennas Propag., vol. 24, no. 6, pp. 841-846, Nov. 1976.

[18] S. Chang, Y.-S. Wang, and S.-J. Chung, "A decoupling technique for increasing the port isolation between strongly coupled antennas," IEEE Trans. Antennas Propag., vol. 56, no. 12, pp. 3650-3658, Dec. 2008.

[19] C.-Y. Lui, Y.-S. Wang, and S.-J. Chung, "Two nearby dual-band antennas with high port isolation," presented at the IEEE Int. Symp. Antennas Propag., San Diego, CA, USA, Jul. 2008.

[20] A. Diallo, C. Luxey, P. L. Thuc, R. Staraj, and G. Kossiavas, "Study and reduction of the mutual coupling between two mobile phone PIFAs operating in the DCS1800 and UMTS bands," IEEE Trans. Antennas Propag., vol. 54, no. 11, pp. 3063-3073, Nov. 2006.

[21] C. Luxey, "Design of multi-antenna systems for UTMS mobile phones," in Proc. Loughborough Antennas Propag. Conf., pp. 57-64. Nov. 2009.

[22] F. Yang and Y. R. Samii, "Microstrip antennas integrated with electromagnetic band-gap EBG structures: A low mutual coupling design for array applications,” IEEE Trans. Antennas Propag., vol. 51, no. 10, pp. 2936-2946, Oct. 2003.

[23] C. Y. Chiu, C. H. Cheng, R. D. Murch, and C. R. Rowell, "Reduction of mutual coupling between closely-packed antenna element," IEEE Trans. Antennas Propag., vol. 55, no. 6, pp. 1732-1738, Jun. 2007.

[24] M. M. Bait-Suwailam, M. S. Boybay, and O. M. Ramahi, "Electromagnetic coupling reduction in high-profile monopole antennas using single-negative magnetic metamaterials for MIMO applications," IEEE Trans. Antennas Propag., vol. 58, no. 9, pp. 2894-2902, Sep. 2010.

[25] B. K. Lau and J. B. Andersen, "Simple and efficient decoupling of compact arrays with parasitic scatterers," IEEE Trans. Antennas Propag., vol. 60, no. 2, pp. 464-472, Feb. 2012.

[26] L. Zhao, L. K. Yeung, and K.-L. Wu, "A coupled resonator decoupling network for two-element compact antenna arrays in mobile terminals," IEEE Trans. Antennas Propag., vol. 62, no. 5, pp. 2767-2776, May 2014.

[27] L. Zhao and K.-L. Wu, "A broadband coupled resonator decoupling network for a three-element compact array," in Proc. IEEE MTT-S Int. Microw. Symp. pp. 1-3. Jun. 2013.

[28] L. Zhao, L. K. Yeung, and K. L. Wu, "A novel second-order decoupling network for two-element compact antenna arrays,"Proc. Asia-Pacific Microwave Conf., 2012.

[29] K. Qian, L. Zhao, and Ke-Li Wu, “An LTCC Coupled Resonator Decoupling Network for Two Antennas" IEEE Transactions on Antennas and Propagation, vol. 63, No. 7, July 2015.

[30] M. T. Ivrlac, W. Utschick, and J. A. Nossek, "Fading correlations in wireless MIMO communication systems," IEEE J. Sel. Areas Commun., vol. 21, no. 5, pp. 819-828, June 2003.

[31] D. Piazza, N. J. Kirsch, A. Forenza, R. W. Heath, and K. R. Dandekar, "Design and evaluation of a reconfigurable antenna array for MIMO systems,” IEEE Trans. Antennas Propag., vol. 56, no. 3, pp. 869-881, Mar. 2008.
[32] D. Shiu, G. J.Foschini, M. J. Gans, and J. M. Kahn, "Fading correlation and its effect on the capacity of multielement antenna systems," IEEE Trans. Commun., vol. 48, no. 3, pp. 502-513, Mar. 2000.

[33] C. Wang and R. D. Murch, "Adaptive downlink multi-user MIMO wireless systems for correlated channels with imperfect CSI," IEEE Trans. Wireless Commun., vol. 5, no. 9, pp. 2435-2446, Sep. 2006.

[34] A. M. Tulino, A. Lozano, and S. Verdu, "Impact of antenna correlation on the capacity of multiantenna channels," IEEE Trans. Inf. Theory, vol. 51, no. 7, pp. 2491-2509, July 2005.

[35] G. Alfano, A. M. Tulino, A. Lozano, and S. Verdu, "Capacity of MIMO channels with one-sided correlation,” in Proc. IEEE Conf. Spread Spectrum Techniques and Applications, pp. 515-519, 2004

[36] S. Jin, M. R. McKay, C. Zhong, and K. K. Wong, "Ergodic capacity analysis of amplify-and-forward MIMO dual-hop systems," IEEE Trans. Inf. Theory, vol. 56, no. 5, pp. 2204-2224, May 2010.

[37] S. Jin, M. R. McKay, X. Gao, and I. B. Collings, "MIMO multichannel beamforming: SER and outage using new eigenvalue distributions of complex noncentral Wishart matrices,” IEEE Trans. Commun., vol. 56,no. 3, pp. 424-434, Mar. 2008.

[38] M. Kiessling and J. Speidel, "Analytical performance of MIMO zeroforcing receivers in correlated Rayleigh fading environments," in Proc. Conf. Signal Processing Advances in Wireless Communications, pp. 383-387.

[39] H. Liu, Y. Song, and R. C. Qiu, "The impact of fading correlation on the error performance of MIMO systems over Rayleigh fading channels," IEEE Trans. Wireless Commun., vol. 4, no. 5, pp. 2014-2019, Sept. 2005.

[40] M. Matthaiou, C. Zhong, and T. Ratnarajah, "Novel generic bounds on the sum rate of MIMO ZF receivers," IEEE Trans. Signal Process., vol. 59, no. 9, pp. 4341-4353, Sep. 2011

[41] A. M. Tulino and S. Verdu, "Random matrix theory and wireless communications," Foundations and Trends in Commun. and Inf. Theory, vol. 1, no. 1, pp. 1-182, 2004.

[42] T. Ratnarajah and R. Vaillancourt, "Quadratic forms on complex random matrices and multiple-antenna systems," IEEE Trans. Inf. Theory, vol. 51, no. 8, pp. 2979-2984, Aug. 2005.

[43] T. Ratnarajah, "Topics in complex random matrices and information theory,” Ph.D. thesis, Univ. Ottawa, Ottawa, Canada, 2003.

[44] A. Alexiou and M. Qaddi, "Robust linear precoding to compensate for antenna correlation in orthogonal space-time block coded systems," in Proc. 2004 Sensor Array and Multichannel Signal Processing Workshop, pp. 701-705.

[45] H. R. Bahrami and T. Le-Ngoc, "Precoder design based on the channel correlation matrices," IEEE Trans Wireless Commun., vol. 5, no. 12, pp. 3579-3587, Dec. 2006.

[46] J. Akhtar and D. Gesbert, "Spatial multiplexing over correlated MIMO channels with a closed-form precoder," IEEE Trans Wireless Commun., vol. 4, no. 5, pp. 2400-2409, Sep. 2005.

[47] S. Zhou and G. B. Giannakis, "Optimal transmitter eigen-beamforming and space-time block coding based on channel correlations," IEEE Trans. Inf. Theory, vol. 49, no. 7, pp. 1673-1690, July 2003.

[48] S. A. Jafar, S. Vishwanath, and A. Goldsmith, "Channel capacity and beamforming for multiple transmit and receive antennas with covariance feedback,” in Proc. IEEE International Conference on Communications, vol. 7, pp. 2266-2270, 2001

[49] T. Ratnarajah and A. Manikas, "An H-infinity approach to mitigate the effects of array uncertainties on the MUSIC Algorithm," IEEE Signal Process. Lett., vol. 5, no. 7, pp. 185-188, Jul. 1998.

[50] Kan Zheng, Suling Ou and Xuefeng Yin "Massive MIMO Channel Models: A Survey "International Journal of Antennas and Propagation Vol. 2014, Article ID 848071, June 2014.

[51] C. Masouros, M. Sellathurai, and T. Ratnarajah, "Large-scale MIMO transmitters in fixed physical spaces: the effect of transmit correlation and mutual coupling," IEEE Transactions on Communications, vol. 61, no. 7, pp. 2794-2804, 2013.

[52] B. Clerckx, C. Craeye, D. Vanhoenacker-Janvier, and C.Oestges, "Impact of antenna coupling on $2 \times 2$ MIMO communications," IEEE Transactions on Vehicular Technology, vol. 56, no. 3, pp. 1009-1018, 2007.

[53] C. Masouros, J. Chen, K. Tong, M. Sellathurai, and T. Ratnarajah, "Towards massive-MIMO transmitters: on the effectsof deploying 
increasing antennas in fixed physical space," in Proceedings of the Future Network and Mobile Summit, pp. 1-10, 2013.

[54] H. T. Hui, "A Practical Approach to Compensate for the Mutual Coupling Effect in an Adaptive Dipole Array," IEEE Transactions on Antennas and Propagation, AP-52, pp. 1262-1269, May 2004.
[55] Y. Yu and H.T. Hui "Design of a Mutual Coupling Compensation Network for a Small Receiving Monopole Array" IEEE Trans. On Micro. Theory and Techniques, vol. 59, no. 9, September 2011.J. C. Coetzee and Y. Yu, "Port decoupling for small arrays by means of an eigenmode feed network,” IEEE Trans. Antennas Propag., vol. 6, pp. 1587-1593, Jun. 2008. 